\title{
Network characteristics enabling efficient coordination: A simulation study
}

Citation for published version (APA):

Khan, A., Peeters, R. J. A. P., Thuijsman, F., \& Uyttendaele, P. (2014). Network characteristics enabling efficient coordination: A simulation study. Maastricht University, Graduate School of Business and Economics. GSBE Research Memoranda No. 004 https://doi.org/10.26481/umagsb.2014004

Document status and date:

Published: 01/01/2014

DOI:

10.26481/umagsb.2014004

Document Version:

Publisher's PDF, also known as Version of record

\section{Please check the document version of this publication:}

- A submitted manuscript is the version of the article upon submission and before peer-review. There can be important differences between the submitted version and the official published version of record.

People interested in the research are advised to contact the author for the final version of the publication, or visit the DOI to the publisher's website.

- The final author version and the galley proof are versions of the publication after peer review.

- The final published version features the final layout of the paper including the volume, issue and page numbers.

Link to publication

\footnotetext{
General rights rights.

- You may freely distribute the URL identifying the publication in the public portal. please follow below link for the End User Agreement:

www.umlib.nl/taverne-license

Take down policy

If you believe that this document breaches copyright please contact us at:

repository@maastrichtuniversity.nl

providing details and we will investigate your claim.
}

Copyright and moral rights for the publications made accessible in the public portal are retained by the authors and/or other copyright owners and it is a condition of accessing publications that users recognise and abide by the legal requirements associated with these

- Users may download and print one copy of any publication from the public portal for the purpose of private study or research.

- You may not further distribute the material or use it for any profit-making activity or commercial gain

If the publication is distributed under the terms of Article $25 \mathrm{fa}$ of the Dutch Copyright Act, indicated by the "Taverne" license above, 
Abhimanyu Khan, Ronald Peeters, Frank Thuijsman,

Philippe Uyttendaele

Network Characteristics enabling Efficient

Coordination: A Simulation Study

RM/14/004

\section{GSBE}

Maastricht University School of Business and Economics

Graduate School of Business and Economics

P.O Box 616

NL-6200 MD Maastricht

The Netherlands 


\title{
Network Characteristics enabling Efficient Coordination: A Simulation Study
}

\author{
Abhimanyu Khan* Ronald Peeters ${ }^{\dagger}$ Frank Thuijsman ${ }^{\ddagger}$ Philippe Uyttendaele $^{\S}$
}

February 15, 2014

\begin{abstract}
The primary question in coordination games concerns the possibility of achieving efficient coordination. We consider a situation where individuals from a finite population are randomly matched to play a coordination game. While this interaction is global in the sense that the co-player can be drawn from the entire population, individuals observe the strategies and payoffs of only the direct connections (or neighbors) in their (social) network. The most successful strategy used in the neighborhood is imitated. We study how the network structure influences the dynamic process of achieving efficient coordination. We simulate this coordination game for small-world and scale-free networks and find that segregation is an important factor in determining the possibility of efficient coordination. In addition, a classification tree analysis reveals segregation to be an important variable of the (non)occurrence of efficient coordination.
\end{abstract}

Keywords: coordination, local imitation, global random interaction, networks

JEL classification codes: C73

*Department of Economics, Maastricht University, P.O. Box 616, 6200 MD Maastricht, The Netherlands. Email: a.khan@maastrichtuniversity.nl.

${ }^{\dagger}$ Department of Economics, Maastricht University, P.O. Box 616, 6200 MD Maastricht, The Netherlands. Email: r.peeters@maastrichtuniversity.nl.

${ }^{\ddagger}$ Department of Knowledge Engineering, Maastricht University, P.O. Box 616, 6200 MD Maastricht, The Netherlands. Email: f.thuijsman@maastrichtuniversity.nl.

${ }^{\S}$ Department of Knowledge Engineering, Maastricht University, P.O. Box 616, 6200 MD Maastricht, The Netherlands. Email: philippe.uyttendaele@maastrichtuniversity.nl. 


\section{Introduction}

Networks - social, economic, institutional, or others - are an important determinant of social and economic behavior by governing the manner in which information flows amongst individual units. Network characteristics such as the density of links (or connections), the asymmetric influence that certain individual units may bear on the network, and the segregation of individual units in the network have a direct bearing on social and economic outcomes. The present paper presents a simulation study based on an evolutionary approach in the context of coordination games (or coordination problems). The aim is to analyze and examine important features of a network that determine the standard or convention that a society adopts. Important questions include: does a society always settle on the better (best) convention (or standard)? What are the network features that facilitate coordination on the better convention?

A coordination game is a simple representation of a situation where multiple equilibria exist: for example, a good or preferred equilibrium and a bad or undesirable equilibrium. An archetypical example of coordination games involves technology adoption. When multiple technologies compete, then it is often seen that society would benefit by the adoption of one technology as a standard, as it is more conducive for collaboration amongst individuals. The question is which technology will be adopted? This issue is even more pertinent when one technology is better than the other, but its adoption may be stymied by the absence of a critical mass of users or asymmetries in compatibility between standards. Thus, while use of any technology as a standard represents an equilibrium, one particular equilibrium, described by adopting the technology that is superior is better than the other equilibria, where other technologies are the standard. The determination of the equilibrium that will prevail is a nontrivial question and the process by which individuals obtain information about the current state of the world plays a crucial role. In addition, initial configurations of the network (via path dependence) also have a major say. This paper attempts to undertake an exploratory study into the features of networks and the initial configurations that are influential for the emergent outcome.

We consider a finite group of individuals that form a society. Some individuals are linked to each other via social or other ties. These ties or connections permit individuals to exchange information in an undirected way, i.e. information flows in both directions. In addition to this, each individual is randomly matched to another to interact in a strategic setting described by a coordination game. One can interpret this random matching of two individuals as a situation where two randomly chosen individuals have to interact with the technology as the interface. Each individual can choose between two strategies (e.g., choice of technology), say $P$ and $R$. While it is better to coordinate on strategy $P$ than to coordinate on strategy $R$, in case of mis-coordination it is preferable to choose strategy $R$. 
Individuals choose a strategy, and the payoff for each individual depends on his own strategy and that of the randomly chosen co-player. After an individual receives the payoff, he exchanges information about his experience (i.e. strategy used and payoff received) with other individuals with whom he shares a social connection. On having obtained such information from his social links, in the next period, he chooses to imitate the action of the most successful individual in his social circle. This imitation rule describes how an individual chooses his action in the next period, where he will be matched to another randomly drawn individual. Clearly, the characteristics of the network shape the way in which information exchange takes place, and how individuals choose one strategy over the other. This situation ensues recurrently, and eventually this process will lead to a situation where the entire population coordinates on one particular action. Our interest lies in the possibility of coordinating on the efficient or preferred equilibrium (all play strategy $P$ ). The questions that we ask are: $(1)$ what are the important initial characteristics of the network for coordinating on the efficient equilibrium, and (2) what is their effect on the time needed to settle on this equilibrium.

The situation that we have described is representative of situations where individuals do not have complete knowledge of their environment. Consequently, they are compelled, while choosing the future course of action, to use the limited information available to them. We capture this process of information diffusion (or information exchange) amongst individuals a critical factor in technology adoption - by a (social) network. Individuals may start with a particular technology choice, but their future choice is governed partly by their own experience with it, and partly by the experience of their acquaintances. In this case, we assume that individuals choose the technology that, according to their information, may lead to the most successful experience. The imitation behavior of individuals is meant to encapsulate a ruleof-thumb that individuals use, possibly due to the complexity of the environment. We assume imitation of the most successful observable strategy (as opposed to other imitation dynamics such as imitation of the strategy that does best on average) because a successful strategy is more visible and prominent than, say, a successful-on-average strategy.

To answer the two questions posed above, we conduct a simulation study. For a given population size and average degree, we generate two network types that are relevant for social interactions: scale-free networks and small-world networks (the construction of which we describe later in more detail). The reason behind choosing these two network types is that they resemble empirical data on networks - in scale-free networks, a few individuals are very well connected while most of the individuals are not as well connected, as a result of which the average path length is low and connectivity is low at the same time; in smallworld networks, the distance between two randomly chosen nodes grows proportionally to the logarithm of the number of nodes in the network. ${ }^{1}$ The initial assignment of strategies $(P$

\footnotetext{
${ }^{1}$ Barabási and Albert (1999) find that the way in which web pages in the internet are connected can be described by a scale-free networks. Soramaki et al. (2007) find that the network topology of the interbank
} 
or $R$ ) amongst individuals is done randomly. Initial conditions may be important in shaping the final outcome due to path-dependence, and in order to study their effect, the proportion of individuals who begin with strategy $P$ (or $R$ ) is varied. Then, the matching and imitation procedure described above is carried out until the first instance appears where all individuals choose the same action - here, the society has reached a convention and adopted a particular strategy (or technology) as the standard.

As mentioned earlier, there are two features of this entire process that we intend to develop an understanding of: the relation between the network characteristics and the probability of reaching population-wide efficient coordination, and their influence on the time taken to reach coordination. The factors that may explain these two may be grouped into two categories: factors that arise from network specific characteristics (NSC) and factors that arise due to the initial assignment of strategies (IAS). Examples of NSC factors include the size of the network, the average degree or the average number of links per node (or individuals) in the network (this is indicative of the average connectivity in the network), the standard deviation in the number of links per node (this reflects the disparity in connectivity in the network) and the average centrality of the nodes in the network as given by the positional power. Examples of IAS factors include the share of nodes who are initially seeded with strategy $P$, their average number of links, their centrality and the extent to which they are segregated from others who have initially been assigned strategy $R$. We describe these factors in Section 2, and the effects of these factors in Section 3.

In the context of imitation in coordination games, Robson and Vega-Redondo (1996) study coordination games with global random matching, where individuals imitate the strategy that yields the highest average payoff globally, and find that for a large enough population, efficient coordination is achieved in the long-run. While global interaction is an element of our model, we stress on the "local nature" of individual's decisions by emphasizing the role of networks. There exists a wide theoretical literature on coordination games and networks where the interaction and information are local. In Chen et al. (2013), individuals located on a circle interact with their two neighbors and imitate actions with the highest average payoffs. They find that the inefficient equilibrium is always stochastically stable, and derive conditions when the efficient equilibrium co-exists in the long-run. Noticeably, the interaction

payments transferred between commercial banks resemble scale-free networks - the network includes a tightly connected core of banks to which most other banks connect. Barabási and Bonabeau (2003) present other examples of the occurrence of scale-free networks. On the other hand, Kogut and Walker (2001) examine the network of cross-ownership among German firms and find evidence of it being described by small-world networks. Davis et al. (2003) study the structure of the Fortune 1000 network of corporate directors and the company interlocks of a cohort of 195 Fortune 1000 firms from 1982 to 1999, and come to a similar conclusion. Fleming and Marx (2006) studied inventors in Silicon Valley and Route 128 in Boston, and find that the network of inventors has a small-world structure, while Newman (2004) find the same structure in coauthorship networks in biology, physics and mathematics. Uzzi et al. (2007) discuss other empirically relevant occurrences of small-world networks. 
and information is both local. On the other hand, Alós-Ferrer and Weidenholzer (2008) and Khan (2013) disentangle information and interaction. The former analyzes imitation behavior in coordination games when individuals interact locally but obtain information from outside their neighborhood, and find that large enough population coordinate efficiently. In contrast, the latter assumes that interaction is global but information is locally obtained from the ties in the network, and shows that even though the efficient equilibrium is always stochastically stable, the inefficient equilibrium may co-exist in the long-run. ${ }^{2}$

On the other hand, to the best of our belief, empirical or simulation analyses of issues pertaining to the role and influence on information on the coordination process is less prevalent. Buskens and Snijders (2008) simulate coordination games where individuals play only with their neighbors. They find that the density of the network and the centralization are important factors that promote efficient coordination. In this paper, we adopt the set-up proposed in Khan (2013), as our interest lies in coordination where individuals may not have full information about the environment they operate in - thus individuals interact with others who may be outside their respective neighborhoods, but information comes only from the neighborhood. Khan (2013) shows that in such an environment, sufficient conditions that ensure efficient coordination to be the unique long-run outcome include each individual being linked to at least four other individuals or when each individual observes the same number of other individuals.

The paper is organized as follows. Section 2 describes the simulation methodology. Section 3 contains the results. We conclude with Section 4.

\section{Methodology}

The payoff matrix below describes the coordination game dealt with in this paper.

\begin{tabular}{c|cc}
\multicolumn{1}{c}{} & \multicolumn{1}{c}{$P$} & \multicolumn{1}{c}{$R$} \\
\cline { 2 - 3 }$P$ & $a, a$ & $b, c$ \\
$R$ & $c, b$ & $d, d$ \\
\cline { 2 - 3 } & &
\end{tabular}

The conditions that we impose on the payoffs are: (1) $a>c, d>b,(2) a>d$ and (3) $c>b$. Condition (1) says there are two strict equilibria represented by the strategy pairs $(P, P)$ and $(R, R) ;(2)$ says the equilibrium payoff of coordinating on strategy $P$ dominates the payoff from the other equilibrium; and (3) says that in case of mis-coordination, it is preferable to choose strategy $R$.

Individuals from a finite population are randomly matched to play this coordination game and receive payoffs depending on their own strategy and that of their randomly drawn coplayer. After receiving payoffs, the individuals update their strategy for the next period by

\footnotetext{
${ }^{2}$ Weidenholzer (2010) reviews the theoretical literature on networks and coordination games.
} 
local imitation: each individual has a set of neighbors (this set includes the individual himself) whom he can observe. An individual imitates the strategy that yields the highest payoff in his observation set. This continues recurrently. Khan (2013) shows that this process converges to the state where either all individuals play strategy $P$ or all play $R$ and provides two sufficient conditions (related to the network architecture) for efficient coordination to be the unique long-run outcome - each individual should be linked to at least four other individuals or the network is regular. The former can be interpreted as a condition on the degree of the nodes, while the latter may be interpreted as a condition relating to the variability in links across nodes. Our simulation study aims to extend the scope of these sufficient conditions and to identify characteristics that determine the likelihood of convergence to the efficient equilibrium, and the time needed for this event to occur. Furthermore, we would like to identify the network characteristic that is the most prominent indicator of the emergence of efficient coordination.

In our simulations, we construct scale-free networks and small-world networks using the method developed by Barabási and Albert (1999) and Watts and Strogatz (1998) respectively. For clarity, let us first describe the construction of a small-world network. Suppose we want to construct a network for $n$ nodes (where $n$ takes values 100, 200 and 300) with mean degree equal to $d$ (we choose $d$ to take the values 4,6 and 8 ). To begin with, ll the nodes are arranged along the circumference of a circle and each node is connected to the nearest $\frac{d}{2}$ nodes on either side. Thereafter, each link is rewired with an exogenously chosen probability, $p$, i.e. each of the links above is broken with a certain probability, and connected randomly to another node. The probability of relinking, $p$, takes all values from 0.00 to 1.00 with stepsize 0.10 . This procedure results in small-world networks, named so because the distance between two randomly chosen nodes in a network grows proportionally to the logarithm of the number of nodes in the network. We generate 100 different networks for each triplet $(n, d, p)$ to smoothen the randomness in this process.

Scale-free networks are constructed by the method of preferential attachment as introduced by Barabási and Albert (1999). Suppose we want to construct a network for $n$ nodes and mean degree close to 4 . We begin with three nodes (say $i, j$ and $k$ ), each of which is connected to the other two. Next, we specify that each incoming node will be connected to exactly two other nodes, and the nodes to which this new entrant will be connected vary probabilistically with the degree of the existing nodes. So, the fourth node $(l)$ has equal probability of being connected to each of $i, j$ and $k$ (and $l$ is connected to exactly two of these three nodes), as $i$, $j$ and $k$ have the same number of connections (i.e. two). But now suppose that $l$ is connected to $i$ and $j$. Then the next entrant (node $m$ ) has a higher probability of being connected to $i$ and $j$ (as they have three links) as compared to $k$ and $l$ (who have two links). Entrants enter the network until we have the desired number of nodes in the network. Thus, each entrant would rather connect to a node with a high degree. In order to generate a network that has an 
average degree close to $d$, we begin with $d+1$ nodes that are all connected to each other, and then make each entrant add $\frac{d}{2}$ links to the network, such that the probability of linking to an existing node is in proportion to the degree of the existing nodes. This algorithm generates scale-free networks.

Table 1 below describes our simulation strategy. We consider three population sizes, comprising of 100, 200 and 300 nodes. For each population size, we vary the number of links per node to be 4, 6 or 8 for small-world networks, and approximately 4, 6 or 8 for scale-free networks. ${ }^{3}$ For small-world networks, as the network generation process is probabilistic due stochastic rewiring, for each combination of network size, a particular number of links per node and probability of rewiring, 100 networks are generated; consequently, 9, 900 networks are generated in all. On the other-hand, for scale-free networks, as the network generation process is probabilistic due to preferential attachment, for each combination of network size and number of links to be added per entrant, 100 networks are generated; consequently, 900 networks are generated in all. It is important to remark that the network specific characteristics (NSC, discussed later) are generated at this stage. Once the network is created, it is necessary to seed a fraction of the nodes with strategy $P$ (and the remaining with strategy $R$ ). The table indicates the proportion of nodes seeded with strategy $P$, for each of the 9,900 (900) small-world (scale-free) networks. While the proportion of initial strategy $P$ nodes is fixed at 10 different values, the assignment is random, and we repeat the process of assignment 100 times for each of the proportions in the table. This leads to 9, 900, $000(900,000)$ potentially different initial small-world (scale-free) network configurations. The characteristics that arise from the initial assignment of strategy (IAS, discussed below) are generated at this stage. Each configuration is run 100 times to account for the random matching assignment, leading to $990,000,000(90,000,000)$ simulations for small-world (scale-free) networks.

After each configuration is created, we simulate the process of playing the coordination game under global random interaction and local imitation in the following way. Each node is randomly matched to another node. The strategy combination of the matched nodes yields the payoffs in accordance to the matrix presented earlier. After the matching and payoff realization process is completed, the strategy updating phase begins. Each node looks at the payoffs and strategies of its neighbors based on the network configuration (we assume the node to be a part of its own neighborhood), and adopts the strategy that yielded the highest payoff. After each node has updated its strategy in this manner, the process of random matching continues until we reach a state where all nodes have the same strategy (i.e. a convention has been established) - clearly, there can be no further change in the strategy of a node beyond this point. We collect data on the particular convention established (strategy $P$ or strategy

\footnotetext{
${ }^{3}$ More precisely, each incoming node adds 2, 3 or 4 links to the network, and the average degree of such networks generated is approximately 4,6 or 8 . For more details, we refer to the variable description of "degree" of nodes that follows.
} 


\begin{tabular}{|c|c|c|}
\hline \multirow[b]{2}{*}{ Parameter } & Small-world network & Scale-free network \\
\hline & Values & Values \\
\hline Population size & 100,200 and 300 & 100,200 and 300 \\
\hline Number of links per node & 4,6 and 8 & 4, 6 and 8 (approx.) \\
\hline Relinking probability & $\begin{array}{l}0.00,0.10,0.20,0.30 \\
0.40,0.50,0.60,0.70 \\
0.80,0.90 \text { and } 1.00\end{array}$ & \\
\hline Number of reconfigurations & 100 & 100 \\
\hline Share of nodes seeded with strategy $P$ & $\begin{array}{l}0.02,0.05,0.10,0.15 \\
0.20,0.25,0.30,0.35 \\
0.40 \text { and } 0.45\end{array}$ & $\begin{array}{l}0.02,0.05,0.10,0.15 \\
0.20,0.25,0.30,0.35 \\
0.40 \text { and } 0.45\end{array}$ \\
\hline Number of reconfigurations (per network) & 100 & 100 \\
\hline Number of initial configurations & $9,900,000$ & 900,000 \\
\hline Number of runs per initial configuration & 100 & 100 \\
\hline Total number of runs & $990,000,000$ & $90,000,000$ \\
\hline
\end{tabular}

Table 1: Simulation parameters, number of random drawings and runs for small-world networks and scale-free networks.

$R$ ), and the number of times we have to conduct the random matching until the first instance that all nodes choose the same strategy (the convergence time). As can be seen from Table 1, to account for the randomness in the matching process, for a given network and given initial assignment of strategies, each initial configuration is played out 100 times. The data on the convention established and the convergence time is aggregated at this level, i.e. the convention established indicates the fraction of times in the 100 runs of the random matching process that efficient coordination is achieved for a given network and given initial conditions; while the mean convergence time is the average number of iterations of random matching needed to converge to a convention for a given network and given initial strategy assignments.

Before discussing the results, we define the factors that we consider to have a possible impact on the probability to reach efficient coordination (on $P$ ) and the time taken to achieve this. As mentioned earlier, they can be categorized into factors that arise from the network (NSC) and factors that arise from initial assignment (IAS).

First, the NSC factors. These are:

(i) Size, i.e. the number of nodes. Since we have only three different network sizes, we treat this as a categorical variable, and interpret the networks with 100, 200 and 300 nodes as small, medium and large networks. The medium-sized network, with the number of nodes equal to 200, is the baseline, and we examine the effect of a smaller (larger) population on efficient coordination.

(ii) Density: This is the fraction of links that are actually formed compared to the maximum number of links that can be formed in the network. 
(iii) Degree (mean and standard deviation): This is the average number of links for a node in the network and the standard deviation in the degree of the nodes. For small-world networks, by construction of the network, the average degree of the network takes only three values (i.e. 4, 6 and 8), and we treat this as a categorical variable. Networks where each node has degree 4, 6 and 8 are interpreted as networks with low, medium and high connectivity. On the other hand, for scale-free networks, the average degree of the network where each entrant adds 2 links and the number of nodes is equal to 100, 200 and 300 is $3.94,3.97$ and 3.98 respectively. We group these networks types together and interpret them as networks with low connectivity. Similarly, the average degree of the network where each entrant adds 3 (4) links and the number of nodes is equal to 100, 200 or 300 is $5.88,5.94$ or $5.96(7.80,7.90$ or 7.93$)$ respectively; we label these networks as networks with medium (high) connectivity. The network with medium connectivity is the baseline and we examine the effect of a network with greater (lesser) connectivity on efficient coordination. The standard deviation in node-degree is treated as a continuous variable.

(iv) Power (standard deviation): By the term power we want to capture the effect that being connected to a well-connected node is different from being connected to a node that is not well-connected. It is desirable to consider not only the connectivity of the direct connections but that of indirect connections as well, and this is not taken into account by, say, the degree of a node. To incorporate this feature, we use the long path method, which is a global positional measure of a node (from Wei (1952) and Kendall (1955)). ${ }^{4}$ After constructing this power measure, we look at the standard deviation in power across nodes.

Next, we present the IAS factors. These are:

(i) Share of $P$ nodes: This is the fraction of nodes that are assigned strategy $P$ while setting up the initial conditions.

(ii) Degree of $P$ nodes (mean and standard deviation): This refers to the average degree and the standard deviation in degree of the nodes who have been given strategy $P$ in the initial assignment.

(iii) Power of $P$ nodes (sum, mean and standard deviation): As in (iv) of NSC factors above, we want to look into the impact of nodes being connected to well-connected nodes. From the power statistic we derive the total power of the seed strategy $P$ nodes, their mean power and the standard deviation in power across such nodes.

\footnotetext{
${ }^{4}$ This power measure is equivalent to the highest positive eigenvalue of the neighborhood matrix that can be constructed from the network. One well-known application of power of nodes is the PageRank by Page et al. (1999).
} 
(iv) Segregation of $P$ nodes (normalized): This statistic is used to examine the extent to which the seed strategy $P$ nodes are segregated in the population. For this purpose, we use the measure developed by Ballester and Vorsatz (2010). It is defined as the probability that two randomly chosen nodes meet at the end of the random walk, and is based on the following algorithm: first, two seed strategy $P$ nodes are picked randomly. Next, one of them starts a random walk along the links in the network, and the random walk has a fixed probability of being terminated after each step. ${ }^{5}$ The probability that the random walk terminates at the other seed strategy $P$ node yields the normalized segregation index. This measure is invariant to the population size as the two nodes described in the random walk procedure are chosen randomly, and the termination probability is fixed (i.e. does not vary with population size).

(v) Segregation of $R$ nodes (normalized): We construct a similar measure for seed strategy $R$ nodes.

It is important to note that after the initial conditions are assigned and the process of random matching and local imitation is started, the measures indicated by IAS (i)-(v) will change as the nodes update strategies by local imitation. However, since we are interested in understanding the effect of initial conditions, we only use the measures as generated from the initial network and its initial distribution.

The variables that we want to explain by the above-mentioned factors are:

1. Payoff dominant wins: The proportion of times that for a given network and given set of initial conditions (i.e. holding fixed the network that is generated for a given population size, degree per node, relinking probability and holding fixed the initial assignment of strategies), the population coordinated on the payoff-dominant (or efficient) equilibrium.

2. Conditional mean convergence time: We continue the random matching and local imitation process until the population coordinates on an equilibrium, and the convergence time is given by the number of iterations of the random matching process until convergence. The mean convergence time indicates the average time for coordination (to any equilibrium) for a given network and given its initial conditions (i.e. holding fixed the network that is generated for a given population size, and holding fixed the initial assignment of strategies). Recall that for any given network and any given set of initial conditions, the process of random matching and local imitation goes over 100 runs, and the average time is taken over these 100 runs. Since our interest lies in the time taken to attain efficient coordination, we only examine the mean average time, conditional on the observed proportion of efficient coordination being at least 0.75 .

\footnotetext{
${ }^{5}$ The probability of termination that we use is 0.15 , which implies that the expected number of steps in a random walk is approximately 6 .
} 
We use a linear regression model to identify the factors that influence the local imitation process. After having done so, we attempt to provide a first answer to the question of the relative importance of these factors. In other words, if it were possible to create a hierarchy of factors by their influence on the local imitation process, which factor would be at the top of the pyramid? For this purpose, we use a classification tree algorithm to see if it is possible to predict the outcome of the simulations based on the NSC and IAS measures. A classification tree algorithm is a machine learning technique that builds a decision tree for a given data set. Based on a target value (in our case the choice of the equilibrium), the data set is separated into (two) subsets that assign a different classification to the observations. The classification is based on one of the (NSC or IAS) measures. Above a given value it assigns one particular outcome and below this given value, another. When a subset contains some instances in the data that are mis-classified (the predicted outcome of the classification is wrong), the process is re-iterated to improve the number of well classified instances. When all instances are correctly classified, the process is terminated, leading to a decision tree. ${ }^{6}$

\section{Results}

We split the following discussion into two subsections, one pertaining to the frequency of attaining efficient coordination, and the second pertaining to the time taken to attain efficient coordination. Before going into the results, we address a relevant aspect of the imitation dynamic. Since we impose an imitate-the-best-neighbor dynamic on the individuals, one observation of an individual receiving payoff $a$ (in reference to the payoff matrix) is sufficient to induce imitation of strategy $P$. This is the only event when strategy $P$ will be imitated. On the other hand, imitation of strategy $R$ will occur either when it is the only strategy in the neighborhood, or in case of neighborhoods that have both strategies, the strategy $P$ individuals are matched to strategy $R$ individuals. Thus, for imitation of strategy $R$ in a mixed neighborhood, the random matching process needs to be unfavorable for all strategy $P$ individuals, whereas for imitation of strategy $P$, the matching process needs to be favorable for only one such strategy $P$ individual.

\subsection{Efficient coordination}

The variable Payoff dominant wins captures the proportion of times that convergence to the efficient action is achieved for a given network and a given distribution of initial strategies, while the process of random matching is repeated. The total number of observations is 9,900,000 (900,000) for small-world (scale-free) networks (see Table 1). To analyze the

\footnotetext{
${ }^{6}$ Unfortunately this built tree is not reliable, as it is overfitted to the data, i.e. it is tailor-made for the given data set to have $100 \%$ correctly classified instances. The tree is then pruned until an "appropriate" classification is made.
} 
effect of NSC and IAS factors on the likelihood of efficient coordination, we run an ordinary least squares regression on the dependent variable (Payoff dominant wins) with the factors mentioned in the previous section as the explanatory variables. The result of the analysis is displayed Table 2.

\begin{tabular}{|c|c|c|c|c|}
\hline \multirow[b]{2}{*}{ Variable } & \multicolumn{2}{|c|}{ Small-world network } & \multicolumn{2}{|c|}{ Scale-free network } \\
\hline & Coef. & $\begin{array}{l}\text { Robust } \\
\text { Std. Err. }\end{array}$ & Coef. & $\begin{array}{l}\text { Robust } \\
\text { Std. Err. }\end{array}$ \\
\hline Size 100 & -.018594 & $(.000166)$ & -.052502 & $(.000853)$ \\
\hline Size 300 & .006113 & $(.000145)$ & .019339 & $(.000503)$ \\
\hline DegreeMean 4 & -.108388 & $(.000160)$ & -.044391 & $(.000407)$ \\
\hline DegreeMean 8 & .056573 & $(.000143)$ & .017596 & $(.000436)$ \\
\hline Share of $P$ nodes & 2.714903 & $(.000565)$ & 2.397553 & $(.001748)$ \\
\hline Degree of nodes: stdev & .012908 & $(.000148)$ & & \\
\hline Degree of $P$ nodes: stdev & & & .014391 & $(.000112)$ \\
\hline Power of $P$ nodes: mean & & & 3.910410 & $(.143479)$ \\
\hline Power of $P$ nodes: stdev & 1.468833 & $(.072851)$ & & \\
\hline Segregation (norm.) of $P$ nodes & -.029758 & $(.000102)$ & -.109114 & $(.000632)$ \\
\hline Segregation (norm.) of $R$ nodes & -.662857 & $(.003816)$ & -.151357 & $(.004433)$ \\
\hline Constant & .698200 & $(.003861)$ & .305024 & $(.004809)$ \\
\hline Number of obs. & \multirow{2}{*}{\multicolumn{2}{|c|}{$\begin{array}{c}9,900,000 \\
0.8508\end{array}$}} & \multicolumn{2}{|c|}{900,000} \\
\hline R-squared & & & & \\
\hline
\end{tabular}

Table 2: Factors that influence the likelihood for the process to converge to the efficient equilibrium state in small-world networks and scale-free networks.

We discuss the effect that the above factors (all of which significant) have on the possibility of efficient coordination. ${ }^{7}$

(i) Size of the network: Since we treat size of the network as a categorical variable, with size equal to 200 as the baseline, the negative sign of co-efficient of "Size 100", and a positive sign of the coefficient of "Size 300", indicates that increasing (decreasing) the number of nodes in the network has a positive (negative) effect. (The coefficients of "Size 100" and "Size 300" are significantly different.) One reason is that, for a given proportion of initial strategy $P$ seeds, increasing the number of nodes increases the initial number of strategy $P$ adopters. This increases the probability of two strategy $P$ nodes meeting each other, resulting in them realizing the highest payoff. Since one successful pairing

\footnotetext{
${ }^{7}$ In case of scale-free networks, we do not use the standard deviation in degree of nodes as it correlates highly with the mean degree due to the preferential attachment algorithm used in construction of such networks. A higher average degree in the network implies that, due to preferential attachment, the probability of some nodes having a greater concentration is higher, leading to high standard deviation in degree of nodes. For a similar reason, the variable standard deviation of power of nodes is also highly correlated with the mean degree. This is not an issue for small-world networks as the relinking process is random. The consequence of this is that, while we use standard deviation of degree of nodes and standard deviation of power of nodes for small-world networks, they cannot be used for scale-free networks, where we use standard deviation of degree of strategy $P$ nodes and mean power of strategy $P$ nodes instead.
} 
is enough for all the neighbors to imitate the action, a larger network increases the likelihood to reach efficient coordination.

(ii) Mean degree of the network: Here, the networks with an average degree of 6 is baseline. The negative sign of co-efficient of "DegreeMean 4" and a positive sign of the coefficient of "DegreeMean 8" indicates that a higher (lower) average number of links per node in the network boosts (hinders) the likelihood of converging to the efficient equilibrium. (The coefficients of "DegreeMean 4" and "DegreeMean 8" are significantly different.) This is simply because when nodes are more connected on average, the chance of observing a successful strategy $P$ node increases, and in the imitation dynamic proposed here, it only takes one favorable observation to induces a node to imitate strategy $P$. On the other hand, due to the nature of the imitation process, more links (i.e. access to larger number of observations) make imitation of strategy $R$ more difficult.

(iii) Share of seed strategy $P$ nodes: Increasing the initial share of nodes who use strategy $P$ has a positive effect on efficient coordination by increasing the initial number of strategy $P$ adopters, which in turn increases the chance of two strategy $P$ nodes meeting each other and realizing the highest payoff.

(iv) Standard deviation of degree of nodes (for small-world networks): A possible reason for the positive effect of this variable on efficient coordination may be that when the standard deviation of nodes increase, then some of these nodes are better connected and form "hubs", while other nodes are not as well connected. These hubs are relatively more resistant to strategy $R$ for the reason that a greater number of nodes will now have to be affected simultaneously by an unfavorable matching for the hubs to imitate strategy $R$. On the other hand, the hubs can very rapidly imitate to strategy $P$ by imitation as they observe a higher number of nodes and they need only one successful strategy $P$ individual to imitate. Further, once these hubs imitate strategy $P$, their influence extends over a larger number of nodes, who may then also imitate strategy $P$.

(v) Standard deviation of degree of strategy $P$ nodes (for scale-free networks): The standard deviation in degree of the strategy $P$ nodes has a positive effect, the reason for this being that a higher standard deviation in degree implies formation of hubs, which (for reasons explained above) are more favorable for the spread of strategy $P$.

(vi) Mean power of strategy $P$ nodes (for scale-free networks): The positional power of the strategy $P$ nodes also exerts a positive effect on efficient coordination in scale-free networks. The reason for this is similar to the effect of a higher mean degree. The difference is that a high statistic for this factor implies that the strategy $P$ nodes have better indirect connections, which facilitates the contagion of strategy $P$. 
(vii) Standard deviation of power of nodes (for small-world networks): An increase in the standard deviation of the power measure of nodes has a beneficial effect on efficient coordination. The reason for this may be related to the reason mentioned in (iv) above. As the standard deviation of this statistic increases, some nodes acquire larger positional power while others lose positional power. But it is more difficult to induce the nodes which now have larger positional power to imitate strategy $R$, and this may provide some level of immunity against coordinating on the inefficient equilibrium.

(viii) Normalized segregation index of strategy $P$ nodes: If this segregation index is high, then it implies that a strategy $P$ node has a high probability of meeting another randomly chosen strategy $P$ node on a random walk along the links of the network. This implies that neither the strategy $P$ node (which starts on the random walk) nor its neighbors share many links with strategy $R$ nodes. This signifies that initial strategy $P$ users are not very "visible" to strategy $R$ users, reducing the possibility of efficient coordination.

(ix) Normalized segregation index of strategy $R$ nodes: The reason is similar to the one stated in (viii) above. A high segregation of $R$ nodes implies that $R$ nodes are, in general, not likely to observe (many) $P$ nodes, which locks them into the use of the $R$ strategy.

In order to determine the factor that is "most" important or predictive of the outcome, we ran a classification tree algorithm on the data set of our network simulations. The variables used were: Size of the network, Mean degree of seed $P$ nodes, Standard deviation of the degree of seed $P$ nodes, Standard deviation in the power of seed $P$ nodes, Segregation of $P$ nodes and Segregation of $R$ nodes. ${ }^{8}$ A pruned version of the classification tree is presented in Table 3 for the small-world network and in Table 4 for the scale-free network.

Table 3 shows that, on basis of the entire dataset of the small-world network, the probability of converging to the efficient outcome is $59.9 \%$. When the data is separated on basis of the variable Segregation (norm.) of $P$ nodes at a cutoff value of 1.204 , in one part this probability increases to $86.7 \%$ while for the other part it drops to $12.2 \%$. In the second layer of separation, each part is separated again on basis of the variable Segregation (norm.) of $P$ nodes. Table 3 shows that, for the small-world network, the level of segregation of the nodes that are initially seeded with strategy $P$ is the most important factor in predicting whether coordination to the efficient outcome can be achieved. The second level takes the probability of convergence up to $92.3 \%$ in one class and down to $3.7 \%$ in another.

Table 4 shows this index also to be the most decisive factor for the scale-free network, for which comparable results are obtained. However, in contrast to the small-scale network

\footnotetext{
${ }^{8}$ The share of seed strategy $P$ nodes was removed as it leads to an obvious classification.
} 


\begin{tabular}{|c|c|c|c|}
\hline \multirow[b]{2}{*}{ Selection } & \multirow[b]{2}{*}{ Number } & \multicolumn{2}{|c|}{ Convergence to $P$} \\
\hline & & Mean & (Std. Dev.) \\
\hline Original dataset & $9,900,000$ & .599 & $(.428)$ \\
\hline Segregation (norm.) of $P$ nodes $<1.204$ & $6,348,644$ & .867 & $(.243)$ \\
\hline Segregation (norm.) of $P$ nodes $\geq 1.204$ & $3,551,356$ & .122 & $(.223)$ \\
\hline $\begin{array}{l}\text { Segregation (norm.) of } P \text { nodes }<1.204 \text { and } \\
\text { Segregation (norm.) of } P \text { nodes }<1.127\end{array}$ & $4,988,064$ & .923 & $(.182)$ \\
\hline $\begin{array}{l}\text { Segregation (norm.) of } P \text { nodes }<1.204 \text { and } \\
\quad \text { Segregation (norm.) of } P \text { nodes } \geq 1.127\end{array}$ & $1,360,580$ & .661 & $(.316)$ \\
\hline $\begin{array}{l}\text { Segregation (norm.) of } P \text { nodes } \geq 1.302 \text { and } \\
\quad \text { Segregation (norm.) of } P \text { nodes }<1.327\end{array}$ & $1,005,279$ & .337 & $(.294)$ \\
\hline $\begin{array}{l}\text { Segregation (norm.) of } P \text { nodes } \geq 1.302 \text { and } \\
\quad \text { Segregation (norm.) of } P \text { nodes } \geq 1.327\end{array}$ & $2,546,077$ & .037 & $(.098)$ \\
\hline
\end{tabular}

Table 3: Classification tree of the small-world network.

\begin{tabular}{|c|c|c|c|}
\hline \multirow[b]{2}{*}{ Selection } & \multirow[b]{2}{*}{ Number } & \multicolumn{2}{|c|}{ Convergence to $P$} \\
\hline & & Mean & (Std. Dev.) \\
\hline Original dataset & 900,000 & .637 & $(.407)$ \\
\hline Segregation (norm.) of $P$ nodes $<1.302$ & 698,078 & .776 & $(.328)$ \\
\hline Segregation (norm.) of $P$ nodes $\geq 1.302$ & 201,992 & .155 & $(.259)$ \\
\hline $\begin{array}{l}\text { Segregation (norm.) of } P \text { nodes }<1.302 \text { and } \\
\text { Degree of } P \text { nodes }(\text { std. })<2.6095\end{array}$ & 94,721 & .345 & $(.363)$ \\
\hline $\begin{array}{l}\text { Segregation (norm.) of } P \text { nodes }<1.302 \text { and } \\
\text { Degree of } P \text { nodes (std.) } \geq 2.6095\end{array}$ & 603,357 & .844 & $(.264)$ \\
\hline $\begin{array}{l}\text { Segregation (norm.) of } P \text { nodes } \geq 1.302 \text { and } \\
\quad \text { Segregation (norm.) of } R \text { nodes }<0.9752\end{array}$ & 50,002 & .467 & $(.329)$ \\
\hline $\begin{array}{l}\text { Segregation (norm.) of } P \text { nodes } \geq 1.302 \text { and } \\
\quad \text { Segregation (norm.) of } R \text { nodes } \geq 0.9752\end{array}$ & 151,920 & .053 & $(.106)$ \\
\hline
\end{tabular}

Table 4: Classification tree of the scale-free network.

where this index also was used in the second layer of separation, the standard deviation in the degree of the $P$ nodes and the segregation of the $R$ nodes carry some importance.

\subsection{Time taken to achieve efficient coordination}

The variable that records the mean convergence time does so for both efficient and inefficient coordination. Since our interest lies in the time taken to attain efficient coordination, we exclude from the regression set the observations for which efficient coordination was attained less than three-fourth of the times. Thus, we only use the data where Payoff dominant wins is at least 0.75 . This leaves us with 5,265, 575 (53.19\%) observations for small-world networks, and 501,081 (55.61\%) observations for scale-free networks. We use a linear regression model to estimate the average time taken for efficient coordination, the results of which are presented in Table 5. We discuss the effect of the factors (all of which are significant) that influence the 


\begin{tabular}{|c|c|c|c|c|}
\hline \multirow[b]{2}{*}{ Variable } & \multicolumn{2}{|c|}{ Small-world network } & \multicolumn{2}{|c|}{ Scale-free network } \\
\hline & Coef. & $\begin{array}{l}\text { Robust } \\
\text { Std. Err. }\end{array}$ & Coef. & $\begin{array}{l}\text { Robust } \\
\text { Std. Err. }\end{array}$ \\
\hline Size 100 & .091336 & $(.001514)$ & -1.276585 & $(.006075)$ \\
\hline Size 300 & .021843 & $(.001079)$ & 1.136900 & $(.002419)$ \\
\hline DegreeMean 4 & 1.344045 & $(.001665)$ & .988007 & $(.001444)$ \\
\hline DegreeMean 8 & -.594475 & $(.000933)$ & -.483977 & $(.001010)$ \\
\hline Share of $P$ nodes & -11.159790 & $(.004769)$ & -.032329 & $(.000027)$ \\
\hline Degree of nodes: stdev & -1.070549 & $(.001585)$ & & \\
\hline Degree of $P$ nodes: stdev & & & -.053317 & $(.000462)$ \\
\hline Power of $P$ nodes: mean & & & -18.726130 & $(1.124271)$ \\
\hline Power of $P$ nodes: stdev & -247.282000 & $(.900051)$ & & \\
\hline Segregation (norm.) of $P$ nodes & 5.531019 & $(.014139)$ & .496119 & $(.010822)$ \\
\hline Segregation (norm.) of $R$ nodes & 3.704960 & $(.022408)$ & 1.447586 & $(.019315)$ \\
\hline Constant & -.228171 & $(.034900)$ & 4.141509 & $(.028758)$ \\
\hline Number of obs. & \multicolumn{2}{|c|}{$5,265,575$} & \multicolumn{2}{|c|}{501,081} \\
\hline R-squared & \multicolumn{2}{|c|}{0.7788} & \multicolumn{2}{|c|}{0.9014} \\
\hline
\end{tabular}

Table 5: Factors that influence the conditional speed convergence for small-world networks and scale-free networks (conditional on the efficient equilibrium being reached at least threefourth fraction of the time).

time taken for such coordination.

(i) Size: Compared to the baseline network containing 200 nodes, the effect of both larger and smaller networks is to increase the convergence time for small-world networks. The mean conditional convergence time is least for the network of 200 nodes. This inverted U-shaped relation between size and convergence time may be explained by realizing that there are two effects at play here: firstly, a larger network, ceteris paribus, implies that there are a larger number of seed strategy $P$ individuals, and this is favorable in hastening convergence to the Pareto-efficient coordination state. On the other hand, a larger network by dint of its size, slows down the process of convergence. The net effect of convergence time will depend on the relative strength of these two opposing forces. It appears that the first force is stronger as we jump from networks of size 100 to 200 , but as we increase the number of nodes even more, the latter force tends to predominate. However, for scale-free networks, the effect of larger (smaller) networks is to increase (reduce) the convergence time. Here, the second effect mentioned above plays the dominant role, i.e. more time is needed for a larger network as strategy $P$ has to spread in a larger population.

(ii) Mean degree of nodes: If the nodes are better connected on average, then due to a greater number of observations on average and the nature of the imitation process, strategy $P$ can spread faster in the population, while at the same time making it more difficult for strategy $R$ to spread. 
(iii) Share of seed strategy $P$ nodes: A greater share of initial adopters drives faster convergence.

(iv) Standard deviation of degree of nodes (for small-world networks): Higher standard deviation in degree of the nodes (thereby implying that some nodes have greater connectivity at the expense of others) reduces the conditional convergence time. A possible reason for the positive effect of this variable on the time taken to achieve efficient coordination may be that when the standard deviation of nodes increase, then some of these nodes are better connected and form "hubs", while other nodes are not as well connected. For reasons alluded to earlier, these hubs are relatively more difficult to change to strategy $R$, and at the same time, more susceptible to strategy $P$. Additionally, they can more easily induce other nodes to imitate strategy $P$.

(v) Standard deviation of degree of strategy $P$ nodes (for scale-free networks): The positive effect of the standard deviation of strategy $P$ nodes is the same as the reason in (iv) above. A higher standard deviation implies that some strategy $P$ nodes are better connected than others and form hubs. These hubs, due to wider observation span, are more susceptible to strategy $P$ imitation, and then can more easily influence a large number of other (connected) nodes.

(vi) Mean power of strategy $P$ nodes (for scale-free networks): This factor has a positive effect on the process of efficient coordination. When the strategy $P$ nodes have more positional power, they are in a better position to directly or indirectly able to access highly connected nodes, and this expedites efficient coordination.

(vii) Standard deviation of power of $P$ nodes (for small-world networks): This factor reduces the convergence time, and the channel via which it operates is similar to (v) above. Since some nodes have more positional power, they are in a position to be more easily affected and also affect other nodes to strategy $P$.

(viii) Segregation of seed strategy $P$ nodes: As strategy $P$ nodes are less visible to strategy $R$ nodes, the convergence takes longer.

(ix) Segregation of seed strategy $R$ nodes: The reason is that a high segregation of $R$ nodes locks many of the $R$ nodes into the use of the $R$ strategy.

\section{Concluding remarks}

The primary question in coordination games with multiple pure strategy Nash equilibria concerns the possibility of achieving efficient coordination. We consider this question in an environment where individuals are not aware of the entire state of the world. This is 
modeled by embedding a coordination game in a set-up where individuals randomly play the coordination game with another individual from the population and imitate the most successful strategy in their neighborhood. We identify a few important determinants of the emergent equilibrium behavior. Apart from the size of the network and the initial share of adopters of the strategy of the efficient equilibrium, some important factors that give the system a push in the direction of efficient coordination are the mean degree of the network, the standard deviation in the degree of initial adopters in the network, and the standard deviation in power of these initial adopters. On the other hand, segregation of the initial adopters impedes efficient coordination. Conditional on achieving efficient coordination, we find that the average degree and share of the initial adopters of the efficient equilibrium strategy make the convergence to this equilibrium faster. Factors that delay this include the initial segregation of nodes. A point to note is the large degree of commonality in factors that drive or impede the coordination process in both small-world and scale-free networks.

Given the results, one may ask how this process can be influenced so as to increase the likelihood of efficient coordination? In context of the classification tree, segregation comes across as a factor of particular importance. We have seen that segregation of the seed nodes of the two strategies not only hinders efficient coordination, but also slows down the process of convergence to the efficient equilibrium when such an event occurs. A decrease in the segregation index makes for smoother flow of information across the population. Therefore, by promoting measures that reduce segregation of the population, one may gain the twin benefits of a greater likelihood of efficient coordination on the desired equilibrium, and this happens at more rapid pace.

We conclude with a speculation that, apart from the local imitation dynamic, it is the global nature of interaction that gives added importance to segregation. Consider a network with low levels of segregation where the average (or representative) initial strategy $P$ node is in a predominantly strategy $R$ neighborhood. Now, on one extreme, if interaction were local and restricted to the neighborhood, then the strategy $P$ node interacts predominantly with strategy $R$ nodes, and receives a low payoff. Strategy $R$ nodes would relatively rarely observe a successful strategy $P$ node and would not imitate strategy $P$. In addition, the strategy $P$ node would imitate strategy $R$. It seems that the network would more easily converge to the inefficient equilibrium. Global interaction attaches a positive probability to the event that two strategy $P$ nodes will interact, even if they are in a predominantly strategy $R$ neighborhood. This successful experience would then be observed by strategy $R$ nodes, which then locally imitate strategy $P$. Thus, low segregation with local interaction may annihilate strategy $P$ relatively more easy, whereas global interaction in combination with the local imitation dynamic may result in a higher observance of efficiently coordinated networks. 


\section{References}

1. Alós-Ferrer C and Weidenholzer S (2008). Contagion and efficiency. Journal of Economic Theory 143 (1): 251-274.

2. Ballester PC and Vorsatz M (2010). Random-walk-based segregation measures. Fedea Working Paper 2010-30. Forthcoming Review of Economics and Statistics.

3. Barabási A and Albert R (1999). Emergence of scaling in random networks. Science 286 (5439): 509-512.

4. Barabási A and Bonabeau E (2003). Scale-free networks. Scientific American 288 (5): 60-69.

5. Buskens V and Snijders C (2008). Effects of network characteristics on reaching the payoff-dominant equilibrium in coordination games: A simulation study. ISCORE Discussion paper.

6. Chen H-C, Chow Y and Wu L-C (2013). Imitation, local interaction, and coordination. International Journal of Game Theory 42 (4): 1041-1057.

7. Davis GF, Yoo M and Baker W (2003). The small world of the American corporate elite: 1982-2001. Strategic Organization 1 (3): 301-326.

8. Fleming L and Marx M (2006). Managing creativity in small worlds. California Management Review 48 (4): 6-27.

9. Kendall MG (1955) Further contributions to the theory of paired comparisons. Biometrics 11 (1): 43-62.

10. Khan A (2013). Coordination under global random interaction and local imitation. GSBE Research Memorandum 13/004. Forthcoming International Journal of Game Theory.

11. Kogut B and Walker G (2001). The small world of German corporate networks in the global economy. American Sociological Review 66 (3): 317-335.

12. Newman MEJ (2004). Who is the best connected scientist? A study of scientific coauthorship networks. In E Ben-Naim, H Frauenfelder and Z Toroczkai (eds.) Complex networks. Berlin: Springer: 337-370.

13. Page L, Brin S, Motwani R and Winograd T (1999). The PageRank citation ranking: bringing order to the web. Technical Report. Stanford InfoLab. 
14. Robson A and Vega-Redondo F (1996). Efficient equilibrium selection in evolutionary games with random matching. Journal of Economic Theory 70 (1): 65-92.

15. Soramaki K, Bech ML, Arnold J, Glass RJ and Beyeler WE (2007). The topology of interbank payment flows. Physica A 1 (1): 317-333.

16. Uzzi B, Amaral LAN and Reed-Tsochas F (2007). Small-world networks and management science research: A review. European Management Review 4 (2): 77-91.

17. Watts DJ and Strogatz SH (1998). Collective dynamics of 'small-world' networks. Nature 393 (6684): 440-442.

18. Wei T (1952). The algebraic foundation of ranking theory. Ph.D. Thesis, Cambridge University, Cambridge, UK.

19. Weidenholzer S (2010). Coordination games and local interactions: A survey of the game theoretic literature. Games 1 (4): 551-585. 